\title{
English Teaching Design and Application based on Flipped Classroom and micro-blog information diffusion Mode
}

\author{
Jing Yang \\ Zhengzhou university of industrial technology.Henan.China
}

Keywords: Flipped and micro-blog information diffusion;Personalized learning;Teaching mode

\begin{abstract}
The Flipped and micro-blog information diffusion Classroom mode is a new teaching mode which reflects the 'students-centered' principle, reverses the order in which learning content is spread and internalized and makes students be able to allocate cognitive resources so as to obtain good learning effect. However, domestic researches on the Flipped and micro-blog information diffusion Classroom mode still stay at an initial stage. Therefore, in teaching application, educators need integrate advanced teaching concepts and techniques effectively, drive teaching reform and provide personalized learning environment for students.it is essential to enhance cognition and comprehension about the Flipped and micro-blog information diffusion Classroom and rethink about whether domestic education needs the Flipped and micro-blog information diffusion Classroom or not in order to construct localized development paths of the Flipped and micro-blog information diffusion Classroom teaching mode.
\end{abstract}

\section{INTRODUCTION}

It was in the early 19th century that the Flipped and micro-blog information diffusion Classroom thought appeared. In detail, The United States Military Academy at West Point created a set of teaching methods, i.e., students utilized sources provided by their teachers to learn before class, while classroom time was used for group cooperation to solve problems jointly. This teaching method reflected by basic concept of the Flipped and micro-blog information diffusion Classroom. In 2000, Americans Glenn Platt and Maureen Lage introduced a new teaching method while giving lessons at University of Miami, i.e., multimedia and World-Wide-Web were utilized to make students watch teaching videos at home and appointed work was finished by group cooperation in classroom. This teaching method was rudiment of the Flipped and micro-blog information diffusion Classroom, but a related specific term was not given to such a teaching form at that time. In 2001, the situation that Massachusetts Institute of Technology developed 'open courseware projects' drives researches on open educational resources and laid a foundation for application of the Flipped and micro-blog information diffusion Classroom mode. In 2004, the American people Salam Khan made videos about coaching materials and uploaded them to website, which was popular among learners. Later, he founded Khan Research Institution and uploaded more learning materials to network, driving rapid development of the Flipped and micro-blog information diffusion Classroom. In 2007, Jon Bergmann and Aaron Sams, two chemistry teachers at America high schools, uploaded PowerPoint they had made to network and asked students to learn after class and finish homework in classroom. micro-blog social network,referred to as OSNs, is a real-time micro-blog social network ,arise after the popularity of the internet application, such as Facebook, twitter, Sina micro-blog etc..Different users purpose with micro-blog social networks are not same, mainly has the access to information, entertainment, discussion topics, making friends etc..Because of these micro-blog social networks attract the large number of users, its have hundreds of millions of commercial value[5], at the same time, the reason of existence and exponential growth of micro-blog social networks was the lots of information provided by the users, from the angle of the enterprise to say, a single marketing information achieve to large scale diffusion must rely on bigger scale and users flow of micro-blog social network, such as user will share an information from an micro-blog social network to another social network, and different micro-blog social network users are have different business value to enterprises, such as some influential users and stars, these kind 
of users may be more effective to affect its fans to forward the already released information, On the other hand, for some ordinary users, they may not be all the time in micro-blog social networks, their social influence is much weaker than star users, so enterprises choose some star users to publish some commodity marketing information is more advantageous clearly ,However, compare with the micro-blog ocial network scale, the number of a star user followers is still small, if they want to make information diffusion in large range, it needs more users to participate in the information diffusion process obviously.

\section{Teaching design about the Flipped and micro-blog information diffusion Classroom mode}

Making teaching videos. When teachers make videos for students, they should pay attention to the following aspects: it is essential to ensure videos they make accord with requirements of teaching content; videos may adopt the method that combines blackboard writing with sound and time length should be kept within 10 minutes as much as possible; to ensure videos can guide students, teachers should design about elements, such as highlight themes, key points and interaction etc.Setting exercises before class. When teachers set exercises before class for students, they should learn and master students' cognitive structure and design difficult and quantity of exercises reasonably. In addition to ensuring moderate difficult, the number of exercises should not be too large. Teachers should make students feel challenges from exercises, which can contribute to arousing their interest in study.Students watch teaching videos. In this process, students may start with improving learning efficiency and control watching time and rhythm. In another word, they may speed or speed reverse videos or suspend them for thinking and record achievements after watching videos in order that they can communicate and share with other classmates.Students' exercises before class. After watching videos, students need finish exercises that teachers have arranged in advanced to consolidate the knowledge they have learned. In this process, students may think about problems they find. In case such problems cannot be solved, they should write them down in order that they can solve it by communication and interaction at next step.Using the media for mutual communication. In this process, students may have the help of media tools like chat room and message board to communicate with other students, share their achievements in learning, discuss problems they have met in exercises and solve such problems mutually. For the problems that they cannot solve via cooperation, they may tell teachers via remote feedback. At this moment, teachers may help students solve puzzling problems. Since students need finish all links of pre-class activities, parents should supervise students when they learn and coach them when students face with problems to improve efficiency. For students with pool self-control ability, parents need coordinate with teachers for formulate some measures to drive them to learn knowledge better and enhance their comprehension about their children's study in this process.

\section{Teaching design about the micro-blog information diffusion mode}

There are rich research achievement about information diffusion, the research value is mainly manifest in viral marketing,control market rumors spread,micro-blog advertising and so on.The domestic and foreign literature shows that research mainly carry out theory model,linear threshold model, independent cascade model, the game theory model and infectious disease model, to study the subject, and research question focused on maximization information diffusion and minimization negative information diffusion, immunization strategy; network structure how to influence information diffusion and other aspects. Applied theory model to research information diffusion based on social network generally and less use real information diffusion data as the research object.The mechanism of information diffusion and the spread of infectious diseases is similar, infectious diseases spread through contact by person to person , but information is spread through communication by person to person. A lot of studies about information diffusion were based on existing research results of the spread of infectious diseases can be thoroughly. The most widely used model is the SIR model and SIS model,first proposed by Kermack and McKendrick respectively in 1927 and 1932. The SIS model divided people into two groups,susceptible state with 
S and infected state I ,respectively. When susceptible people contact with infected people, susceptible people will be infected with probability $\beta$, and assumed that infected people become to susceptible with probability $\gamma$. The SIR model divided people into three states, susceptible S, infected I and recovery R, similar to the SIS model, when susceptible people contact with infected people, they become infected with probability $\beta$, and infected people become to recovery state with probability $\gamma$. When people become to recovery state and assume that they have permanent immunity and no possibility become to susceptible state again. Figure 1 show the SIS model and SIR model , respectively.
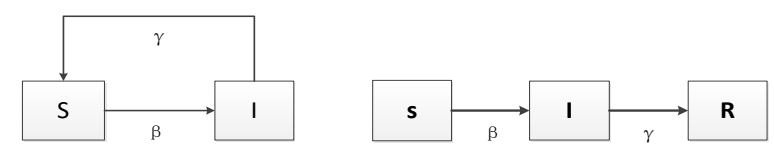

Linear threshold model

Fig.1. The Model of SIS

For each node $\mathrm{v}$ in network, The weight sum of all the edge that connected with node $\mathrm{v}$ need meet the condition $\Sigma_{u \in \nabla} p_{u, v} \leq 1, \nabla$ present the neighbor node set of node v.At the start, part nodes were activated, for any one node, each node was assigned a threshold $\theta_{v}$ between 0 and 1 .At time scale t,a non-activated node $\mathrm{v}$ will affected by all activated neighbors of node $\mathrm{v}$,if the sum of influence

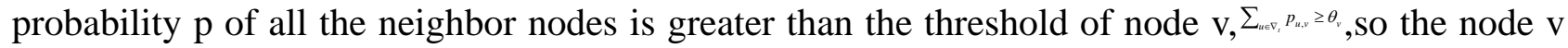
will be activated at time scale $t$,and become activated state at time scale $t+1$, until there is no activated behavior happen and the activated process is terminate.

Independent cascade model

At time scale t,each activated node will activate its neighbor nodes at a probability,if a non-activated node is the neighbor of many activated nodes,these nodes will activate non-activated nodes in order ,and each activated node activate non-activated nodes only once,whether to activate success,activated nodes no longer to activate these neighbors again,if the neighbor node was success activated,it will activate its neighbor node at time scale $\mathrm{t}+1$.

Game theoretical model

Whether a node accept information decided from the angle of benefit maximization of itself,the node will compare social benefit with personal benefit to determine itself maximal benefit.Node make the same choice as its neighbors, it will gain social benefit,but personal benefit is that accept information can bring benefit to people. Few existed research results applied game theoretical to study information diffusion in micro-blog social network.

\section{RESEARCH ON INFORMATION DIFFUSION BASED ON EPIDEMIC MODEL}

Research information diffusion in micro-blog social networks based on epidemic model is mainly from the following several aspects: (1) In addition to susceptible state, infected state and recovery state,some research extended SIS model and SIR model such as increased a new state for users in social networks. Zhao,LJ introduced remembering mechanisms to research a rumor spreading model, Susceptible-Infected-Hibernator-Removed (SIHR) model, the model extends the classical Susceptible-Infected-Removed (SIR) rumor spreading model by adding a direct link from ignorants to stiflers and a new kind of people-hibernators.They discussed the spreading threshold and find the relationship between the final size of the rumor and two probabilities.Numerical simulation shows that the direct link from the ignorants to the stiflers advances the rumor terminal time and reduces the maximum rumor influence. Moreover, the forgetting and remembering mechanisms of hibernators postpone the rumor terminal time and reduce the maximum rumor influence.Zhang, $\mathrm{N}$ considered official rumor refutation and divided people into eight states:ignorance,ignorant removal, rumor carrier,rumor spreader,rumor advocate,truth carrier,truth spreader and truth advocate,and researched relevant factors with rumor spreading and then proposed a new spread model-ICSAR model,and put forward effective methods according to the different situation how to stop the spread 
of rumors.Xiong Fei through described the characteristics of micro-blog information diffusion and based SIR model to propose a SCIR model which have four states, S,I , R and C expressed contact state, and state $C$ denoted that when people of state $S$ contact with people of state I,the group of $S$ state will not change into I state immediately but come into state C,after that people of state C may lost interest to spread information and change into state $\mathrm{R}$ with a certain probability,at the same time, remaining group of state $\mathrm{C}$ change into state I with a certain probability.The results show that even a small infection rate,information may still diffused in scale-free networks with a wide range,and infection individual density is closely related to the average degree of network, even if network average degree is big,there are still exist individual of state $\mathrm{R}$ in network.LvLinyuan study found that many infectious disease transmission dynamics and information diffusion kinetics were had unified frameworks and similarity models, she put forward a new UKAE model that emphasizes information diffusion and disease spreading is different in the essence,and considered the memory effect,social strengthening effect and non- redundant factors in the model.The research results show that the speed and breadth of information diffusion in the rule network is much better than the random network, but it is most efficient in small world network.

(2)Effect of immunization strategy on information diffusion

Many scholars have been studied information diffusion from the perspective of immune in detail, PASTOR proposed the target immunization strategy based on SIS model, that is immune to the nodes in the network which degrees are big. Zhang HF according the immunity of susceptible people and infectious people to classify them to different group, and assumed that the spread of the disease may have more than one stage and modeled to better explained the spread of infectious diseases. Huang,JY made the improvement to the SIR model and then proposed ISRARU model, the research take random immunization strategy and target immunization strategy to prevent rumors spreading in the small world network, and the results find that when the average degree of network is small, two strategies are effective, but when the average degree of network is large, two strategies are invalid. So authors presented a new strategy to reduce the credibility of rumors, and then applied these kinds of immune strategy to compared research and found that even the average degree of network is big, the new strategy has a good effect on prevent rumors spreading.Nian,FZ proposed a kind of effective immune strategy which is a high risk immunization strategy, he believes that the simple immune strategy is object to susceptible individuals which neighbors have been infected, these susceptible individuals are referred to as high risk individuals, correspondingly, authors improved the SIR model based on small world network and scale-free network and the results showed that the high risk immunization strategy was economical and feasible in practice.

(3)User's behavior

Some scholars considered the factors impact on information diffusion, such as user's behavior, because of these studies are relatively less at present, Suqiang taking into account the part of the user's behavior which them not all read micro-blog content, so he based on the SIR model to propose a Mb-SIR mode and expand the content of the model. He extracted data from the Sina micro-blog to verify the model validity, the research results showed that the model has good accuracy, at the same time, compared to the transmission rate and removal rate,reading rate has greater impact on information diffusion. Yan,Q considered the impact of user burst behavior and limited attention on information diffusion in the micro-blog social network, and based these two points to improve the SI model, the research results verified that the effect of information diffusion in micro-blog social networks can be optimized and controlled.

(4)Impact of the social network structure

The research scope of the information diffusion characteristics are the influence factors of information diffusion and the effects of network structure on information diffusion generally, a lot of research in this field are based on the diffusion model.A part of the study focuses on the influence factors, such as the infection probability, memory mechanism, forgetting mechanism, immune strategy and so on. FuXinchu studied the effects of nonlinear infection probability in the SIS model on infectious disease diffusion threshold, this study considered the infection probability between nodes is a non-linear function and different from many studies assumed that the infection 
probability is a variable. Another part of the study mainly focuses on these problems from the perspective of network structure.Mina Youssef believes that previous research had only considered the representative characteristics of network structure, without considered all the network structure details, and also not considered each individual state in the network, many previous studies were assumed susceptible individual turn into infected individual with the same probability, but this study combined the continuous Markov chain with SIR model and considered the infection probability of each individual is different. Chuxiangwei considered the factor of nonlinear infection rate and studied the effects of infection rate index and the weighted index on the disease diffusion threshold and the disease prevalence based on the SIR model in weighted scale-free network, the results showed that the infection rate index had a positive effect on the threshold and the prevalence.

\section{Conclusion}

Under the Flipped Classroom and micro-blog information diffusion mode, it is essential to improve traditional teaching evaluation methods and make teaching evaluation not only cover assessment on several kinds of ability, such as students' cooperative ability, self-study ability, organizing ability and ability of expression but also attaches importance to evaluation on students' emotion, attitudes and value. Besides, teaching evaluation should be finished by learning partners, students themselves, teachers and parents.

\section{Reference}

[1] Zhao Xinglong. Knowledge internalization process and teaching mode design in Flipped Classroom [J]. Modern Distance Education Research, 2014(3).

[2] Lai Yan, Hou Yongxian, Zhao Jiaojiao. The first exploration on Flipped Classroom: application of micro classroom to mathematics at primary schools [J]. Information Technology Education of Middle and Primary School, 2014(3).

[3] Li Cou, Liu Ganhong. SWOT analysis of application of Flipped Classroom teaching mode [J]. Chinese Educational Technology Equipment, 2013(1).

[4]M. Stelzner, Social Media Marketing Industry Report 2009,URL:http://marketingwhitepapers.s3 [5]Hoffman,D.L, Novak,T P.'Toward a Deeper Understanding of Social Media”,Journal of Interactive Marketing,vol.26, pp.69-70,February 2012.

[6]Q.Yan,L.Wu,C.Liu,”Information Propagation in Online Social Network Based on Human Dynamics”,Abstract and Applied Analysis,2013 1-6.

[7]Howard,”Analyzing online social networks”,Communications of the ACM 51 (11),2008:14-16.

[8]H. Oinas-Kukkonen, K. Lyytinen, Y. Yoo”Social networks and information systems: ongoing and future research streams",Journal of the Association for Information Systems, vol.11,pp.6168,February 2010. 